\title{
Initial convergence of the perturbation series expansion for vibrational nonlinear optical properties
}

\author{
Miquel Torrent-Sucarrat, Miquel Solà, Miquel Duran, and Josep M. Luis ${ }^{a)}$ \\ Institute of Computational Chemistry and Department of Chemistry, University of Girona, \\ Campus de Montilivi, 17071 Girona, Catalonia, Spain \\ Bernard Kirtman \\ Department of Chemistry and Biochemistry, University of California, Santa Barbara, California 93106
}

(Received 23 October 2001; accepted 7 January 2002)

\begin{abstract}
$A b$ initio Hartree-Fock and MP2 calculations of the longitudinal (hyper)polarizability-including the static electronic, static zero-point vibrational average (ZPVA), and pure vibrational (static and dynamic) contributions - have been carried out on a set of seven typical medium size conjugated nonlinear optical (NLO) molecules. The ZPVA is obtained through first-order in mechanical plus electrical anharmonicity. Based on physical "nuclear relaxation" considerations the individual (square bracket) terms that contribute to the pure vibrational (hyper)polarizability are then taken into account through third-, fourth-, or fifth-order depending upon the type of term. In order to carry out the correlated treatment, field-induced coordinates and a special finite field technique are utilized. Correlation leads to very substantial differences in the absolute and relative values of the various contributions. In comparison to the electronic term the ZPVA correction is usually small but in one case is over two-thirds as large. On the other hand, both static and dynamic pure vibrational contributions are commonly of a magnitude that is comparable to, or are larger than, the electronic term. The higher-order pure vibration terms are often large. For dynamic processes they can be almost as important as the lowest-order terms; for static (hyper)polarizabilities they can be more important. Thus, for typical NLO molecules, the initial convergence behavior of the perturbation series in mechanical and electrical anharmonicity requires further investigation. (c) 2002 American Institute of Physics. [DOI: 10.1063/1.1453953]
\end{abstract}

\section{INTRODUCTION}

During the last two decades substantial experimental and theoretical research efforts have been devoted to nonlinear optical (NLO) properties of molecular and solid state materials. Recently, attention has focused on $\pi$-conjugated organic systems which are easily synthesized and chemically modified, resist high intensity radiation, and have large nonlinear optical properties in a wide frequency range. At the microscopic level NLO properties are determined by the first and second hyperpolarizabilities, $\beta\left(-\omega_{\sigma} ; \omega_{1}, \omega_{2}\right)$ and $\gamma\left(-\omega_{\sigma} ; \omega_{1}, \omega_{2}, \omega_{3}\right)$. Both electronic and nuclear motions contribute to these properties. Although in the past the latter have often been ignored, it is now well-recognized that the effect of nuclear motions can be of major import. In fact, in many cases vibrational hyperpolarizability contributions are as large as, or larger than, their electronic counterparts. ${ }^{1-7}$

A general sum over states (SOS) perturbation treatment of vibrational hyperpolarizabilities at nonresonant frequencies has been developed by Bishop and Kirtman (BK). ${ }^{8-11}$ In their treatment the pure vibrational component (as distinct from the vibrational averaging contribution) is identified as the set of terms involving one or more intermediate states associated with nuclear motion on the ground electronic state potential-energy surface. These terms are then evaluated by

\footnotetext{
a) Author to whom correspondence should be addressed. Electronic mail: josepm@iqc.udg.es
}

applying a clamped nucleus approximation, ${ }^{12}$ that leads to a complete separation of electronic and pure vibrational contributions. The resulting formulas, expressed in terms of electrical property derivatives with respect to nuclear displacements, can be grouped into various "square bracket" types. Thus, for example, $\beta_{\alpha \beta \gamma}^{\nu}\left(-\omega_{\sigma} ; \omega_{1}, \omega_{2}\right)$ is written as $[\mu \alpha]+\left[\mu^{3}\right],{ }^{1,2}$ where $[\mu \alpha]$ contains products of an electric dipole and a linear polarizability derivative whereas $\left[\mu^{3}\right]$ involves products of three electric dipole derivatives. (The superscript $\nu$ is used here, and henceforth, to denote the pure vibrational component of the hyperpolarizability.) Following the BK treatment, each square bracket is a sum of perturbation terms of different order in electrical and/or mechanical anharmonicity, e.g.,

$$
\begin{aligned}
{[\mu \alpha] } & =[\mu \alpha]^{0,0}+[\mu \alpha]^{2,0}+[\mu \alpha]^{1,1}+[\mu \alpha]^{0,2}+\cdots \\
& =[\mu \alpha]^{0}+[\mu \alpha]^{\mathrm{II}}+\cdots
\end{aligned}
$$

In Eq. (1) the first superscript refers to the order in electrical anharmonicity while the second denotes the order in mechanical anharmonicity; Roman superscripts in the second line give the overall order. The order in electrical anharmonicity is determined by the total order of the electrical property derivatives (first derivatives $=$ order 0 , second derivatives $=$ order 1 , etc.). For mechanical anharmonicity the order is determined by the vibrational force constants or, equivalently, by the vibrational potential-energy derivatives (first derivatives vanish; second derivatives $=$ order 0 ; third 
derivatives $=$ order 1 ; etc.). Compact expressions in terms of these force constants and the electrical property derivatives are given in Ref. 11. For each square bracket type, the leading term may be of overall order 0, I, or II but, in any event, either all odd orders or all even orders will vanish.

The initial convergence behavior of the BK double perturbation series has not been extensively studied. One obvious choice for monitoring initial convergence is the total order. However, for planar $\pi$-conjugated oligomers, where anharmonicity plays a major role in determining $\beta^{\nu}(0 ; 0,0)$, $\gamma^{\nu}(0 ; 0,0,0)$, and $\gamma^{\nu}(-\omega ; \omega, 0,0)$, it has been found ${ }^{13}$ that terms of order I and II are sometimes larger than the zerothorder harmonic terms. It turns out there is another approach to the calculation of pure vibrational hyperpolarizabilities which suggests an alternative grouping of terms that may be more appropriate for determining initial convergence. This other approach is based upon the change in electronic electrical properties due to the equilibrium geometry change (i.e., "nuclear relaxation") induced by a static external electric field. ${ }^{14}$ Consideration of these field-dependent properties ${ }^{15}$ leads to nuclear relaxation (NR) (hyper)polarizability expressions that contain only the lowest-order BK term of each square bracket type evaluated in the limit where the optical frequencies become infinite (the static fields, of course, remain static). In this sense $\alpha^{\mathrm{nr}}, \beta^{\mathrm{nr}}$, and $\gamma^{\mathrm{nr}}$ constitute the leading contribution to the pure vibrational property. For typical laser optical frequencies, test calculations ${ }^{16-18}$ confirm that replacement by $\omega \rightarrow \infty$ does not lead to a significant loss of accuracy, although significant differences can arise at lower frequencies. ${ }^{18}$ From a computational viewpoint NR (hyper)polarizabilities can be evaluated either by means of analytical formulas ${ }^{19}$ as they are in the BK method, or by numerical finite field (FF) techniques. ${ }^{15,20,21}$ The FF treatment is computationally advantageous especially when used in conjunction with highly correlated electronic structure methods and/or when applied to large systems. ${ }^{22}$

The remaining higher-order square bracket terms (denoted below by C-ZPVA), in combination with the zeropoint vibrational averaging (ZPVA) term, collectively give rise to what has been referred to as the curvature (C) contribution $^{23}$ to the property $P$, i.e., $P^{\mathrm{c}}=P^{\text {zpva }}+P^{\mathrm{c}-\text { zpva }}$. Here $P$ is a generic notation for the (hyper)polarizability associated with any NLO (including static) process. (Note that the pure vibrational property is given by $P^{\mathrm{nr}}+P^{\text {c-zpva }}$.) Starting with the static $P^{\text {zpva }}$, Kirtman, Luis, and Bishop (KLB) ${ }^{23}$ have shown that $P^{\mathrm{c}-\text { zpva }}$ can be calculated (in the infinite optical frequency limit) by an FF procedure that is exactly analogous to the one devised by Bishop, Hasan, and Kirtman $(\mathrm{BHK})^{15}$ to obtain $P^{\mathrm{nr}}$ from the electronic properties $P^{e}$.

On the basis of the BHK/KLB approach it has been suggested that, as far as initial convergence is concerned, one should examine separately two different sequences that combine to give the total property value: ${ }^{24}$

(A) $P^{e},\left[P^{\mathrm{zpva}}\right]^{\mathrm{I}},\left[P^{\mathrm{zpva}}\right]^{\mathrm{III}}, \ldots$

(B) $P^{\mathrm{nr}}, P^{\mathrm{c}-\mathrm{zpva}}(\mathrm{I}), P^{\mathrm{c}-\mathrm{zpva}}(\mathrm{III}), \ldots$

Here $P^{\mathrm{c}-\text {-zpva }}(\mathrm{I})$ is used to indicate the fact that this C-ZPVA term is derived from $\left[P^{z p v a}\right]^{\mathrm{I}}$ which, in turn, is first-order in electrical plus mechanical anharmonicity (even-order ZPVA terms vanish). As discussed above (B) pertains only to the infinite optical frequency limit, but we can easily extend the definition to include frequency-dependence (see further following). In our first investigation on the molecule $\mathrm{NH}_{2}-(\mathrm{CH}=\mathrm{CH})_{3}-\mathrm{NO}_{2}$, the (A) and (B) series were each found to be initially convergent, through order I, for $\left.\quad \beta^{\mathrm{nr}}(0 ; 0,0), \quad \beta^{\mathrm{nr}}(-\omega ; \omega, 0)\right)_{\omega \rightarrow \infty}, \quad \gamma^{\mathrm{nr}}(0 ; 0,0,0)$, $\gamma^{\mathrm{nr}}(-\omega ; \omega, 0,0)_{\omega \rightarrow \infty}$, and $\gamma^{\mathrm{nr}}(-2 \omega ; \omega, \omega, 0)_{\omega \rightarrow \infty} .{ }^{24}$ On the other hand, weakly bound systems with highly anharmonic low-frequency vibrational modes, such as the dimers of $\mathrm{HF}^{25,26}$ and $\mathrm{H}_{2} \mathrm{O}^{25}$ are likely to serve as counter-examples. Although the complete set of terms that contribute to series (B) in first-order has not been evaluated for these dimers, it has been found that some of the individual terms are quite large. Thus, it may well be necessary in cases like these to treat one or more modes "exactly" as previously suggested. ${ }^{26}$ In this paper, however, we concern ourselves instead with extending the study of Ref. 24 so as to test the initial convergence, through $\left[P^{\text {zpva }}\right]^{\mathrm{I}}$ and $P^{\mathrm{c}-z p v a}(\mathrm{I})$, for a more comprehensive sample of typical medium-size conjugated organic NLO molecules. As a matter of convenience we will, henceforth, denote these terms by $P^{\text {zpva }}$ and $P^{\mathrm{c} \text {-zpva }}$ unless otherwise specified.

In order to realize our goal, it is necessary to take advantage of a special set of vibrational coordinates, known as field-induced coordinates (FICs). ${ }^{13,27}$ These FICs are the essential coordinates needed for exact calculation of $P^{\text {nr }}$ and $P^{\text {c-zpva }}$ (infinite optical frequency limit). Most importantly, they are limited in number and, in contrast with normal coordinates, their number does not increase with the size of the system. In addition, FICs can be utilized to simplify computation of the static $P^{\text {zpva }} \cdot{ }^{28}$ Finally, we have just accomplished the generalization to include frequency-dependence (though only static coordinates will be employed here). ${ }^{18}$

Although electron correlation is known to have a large effect on nonlinear response, ${ }^{1}$ it has not often been included in the theoretical investigation of pure vibrational hyperpolarizabilities except for small molecules. There are a few instances where correlation has been taken into account, together with the double harmonic approximation, ${ }^{1,6,29}$ for medium-size organic molecules. However, in only two previous cases ${ }^{22,24}$ have anharmonic effects been considered as well. The MP2 hyperpolarizability results, where available, show that electron correlation significantly decreases the importance of the NR contribution relative to its electronic counterpart. No analogous treatments of the ZPVA and C-ZPVA contributions to the hyperpolarizability have been carried out as far as we know. Thus, the second aim of this paper is to further explore the effect of electron correlation on NR hyperpolarizabilities for medium-size organic molecules and to examine $P^{\text {zpva }}$ and $P^{\mathrm{c}-\mathrm{zpva}}$ in the same vein.

\section{THEORY}

As mentioned in the introduction, analytical formulas for the NR (hyper)polarizabilities can be written in terms of static FICs. ${ }^{13}$ The number of FICs needed is independent of the number of atoms $(N)$ in the molecule and is, generally, far less than $3 \mathrm{~N}-6$, which would be required if normal coor- 
dinates were used. Analytical expressions for the FICs may be derived from the equations that give the normal coordinate displacements induced by a static electric field $\left(Q_{i}^{F}\right){ }^{13}$ These displacements are determined by the stationary equilibrium geometry condition applied to an expansion of the potential energy as a double power series in the field-free normal coordinates, $Q_{i}$, and the static electric-field vector with components $F_{\alpha}, F_{\beta}, \ldots{ }^{14,19}$ An iterative solution yields the static linear $\left(\chi_{1}\right)$ and quadratic, or second-order, FICs $\left(\chi_{2}\right):^{13}$

$$
\begin{aligned}
\chi_{1}^{\alpha}= & \sum_{i=1}^{3 N-6} \frac{\partial Q_{i}^{F}}{\partial F_{\alpha}} Q_{i}=-\sum_{i=1}^{3 N-6} q_{1}^{i, \alpha} Q_{i}, \\
\chi_{2}^{\alpha \beta}= & \frac{1}{2} \sum_{i=1}^{3 N-6} \frac{\partial^{2} Q_{i}^{F}}{\partial F_{\alpha} \partial F_{\beta}} Q_{i} \\
= & \sum_{i=1}^{3 N-6}\left[-q_{2}^{i, \alpha \beta}+\sum_{j=1}^{3 N-6} \frac{\alpha_{21}^{i j, \alpha}}{a_{20}^{i i}} q_{1}^{j, \beta}\right. \\
& \left.-\sum_{j, k=1}^{3 N-6} \frac{3 a_{30}^{i j k}}{2 a_{20}^{i i}} q_{1}^{j, \alpha} q_{1}^{k, \beta}\right] Q_{i},
\end{aligned}
$$

where

$$
\begin{aligned}
a_{n m}^{i, j, \ldots, \alpha, \beta} & =\frac{1}{n ! m !}\left(\frac{\partial^{(n+m)} V\left(Q_{1, \ldots}, Q_{3 N-6}, F_{x}, F_{y}, F_{z}\right)}{\partial Q_{i} \partial Q_{j} \cdots F_{\alpha} \partial F_{\beta \ldots}}\right)_{Q=0, F=0}, \\
q_{1}^{i, \alpha}= & \frac{a_{11}^{i, \alpha}}{2 a_{20}^{i i}}, \\
q_{2}^{i, \alpha \beta} & =\frac{a_{12}^{i, \alpha \beta}}{2 a_{20}^{i i}},
\end{aligned}
$$

and $a_{20}^{i i}$ is the harmonic vibrational force constant. For $n$ $=1$ and $m>0$ the parameters $a_{n m}$ are (harmonic) electrical property first derivatives; for $n>2$ and $m=0$ they are mechanical anharmonic force constants; and for $n>1$ and $m$ $>0$ these parameters characterize the electrical anharmonicity. The second-order FICs $\left(\chi_{2}^{\alpha \beta}\right)$ depend upon the anharmonicity parameters $a_{21}$ and $a_{30}$, which are much more timeconsuming to calculate than the harmonic parameters $a_{11}$, $a_{12}$, and $a_{20}$. However, the harmonic second-order FICs defined by eliminating the anharmonic terms of Eq. (3). ${ }^{13}$

$$
\chi_{2, \mathrm{har}}^{\alpha \beta}=\frac{1}{2} \sum_{i=1}^{3 N-6}\left(\frac{\partial^{2} Q_{i}^{F}}{\partial F_{\alpha} \partial F_{\beta}}\right) \underset{\text { har }}{Q_{i}}=-\sum_{i=1}^{3 N-6} q_{2}^{i, \alpha \beta} Q_{i}
$$

are sufficient for many purposes. Indeed, as demonstrated in Ref. 13, the linear and harmonic second-order FICs are all that is needed to obtain $\alpha_{\alpha \beta}^{\mathrm{nr}}(0 ; 0), \beta_{\alpha \beta \gamma}^{\mathrm{nr}}(0 ; 0,0)$, $\beta_{\alpha \beta \gamma}^{\mathrm{nr}}(-\omega ; \omega, 0)_{\omega \rightarrow \infty}, \quad \gamma_{\alpha \beta \gamma \delta}^{\mathrm{nr}}(-\omega ; \omega, 0,0)_{\omega \rightarrow \infty}, \quad \gamma_{\alpha \beta \gamma \delta}^{\mathrm{nr}}$ $(-2 \omega ; \omega, \omega, 0)_{\omega \rightarrow \infty}$, and $\gamma_{\alpha \beta \gamma \delta}^{\mathrm{nr}}(-\omega ; \omega,-\omega, \omega)_{\omega \rightarrow \infty}$ (where the subscript $\omega \rightarrow \infty$ indicates the infinite optical frequency approximation). In order to calculate $\gamma_{\alpha \beta \gamma \delta}^{\mathrm{nr}}(0 ; 0,0,0)$, on the other hand, anharmonic second-order static FICs are required. For that property the coordinates $\chi_{2}$ in Eq. (3) can, alternatively, be determined through the FF procedure. ${ }^{13}$
Exact expressions for the NR (hyper)polarizabilities (except in the two cases where they are zero, i.e., $\beta_{\alpha \beta \gamma}^{\mathrm{nr}}(-2 \omega ; \omega, \omega)_{\omega \rightarrow \infty}$ and $\left.\gamma_{\alpha \beta \gamma \delta}^{\mathrm{nr}}(-3 \omega ; \omega, \omega, \omega)_{\omega \rightarrow \infty}\right)$ are given below in terms of the FICs: $:^{13,30}$

$\alpha_{\alpha \beta}^{\mathrm{nr}}(0 ; 0)=\frac{1}{2} \sum P_{\alpha \beta} \frac{\partial \mu_{\alpha}}{\partial \chi_{1}^{\beta}} \frac{\partial \chi_{1}^{F}}{\partial F_{\beta}}$,

$\beta_{\alpha \beta \gamma}^{\mathrm{nr}}(0 ; 0,0)=\sum P_{\alpha \beta \gamma}\left[\frac{1}{2} \frac{\partial \alpha_{\alpha \beta}}{\partial \chi_{1}^{\gamma}} \frac{\partial \chi_{1}^{F}}{\partial F_{\gamma}}+\frac{\partial^{2} \mu_{\alpha}}{\partial \chi_{1}^{\beta} \partial \chi_{1}^{\gamma}} \frac{\partial \chi_{1}^{F}}{\partial F_{\beta}} \frac{\partial \chi_{1}^{F}}{\partial F_{\gamma}}\right.$

$$
\left.-\frac{1}{6} \frac{\partial^{3} V}{\partial \chi_{1}^{\alpha} \partial \chi_{1}^{\beta} \partial \chi_{1}^{\gamma}} \frac{\partial \chi_{1}^{F}}{\partial F_{\gamma}} \frac{\partial \chi_{1}^{F}}{\partial F_{\beta}} \frac{\partial \chi_{1}^{F}}{\partial F_{\gamma}}\right] \text {, }
$$

$\beta_{\alpha \beta \gamma}^{\mathrm{nr}}(-\omega ; \omega, 0)_{\omega \rightarrow \infty}=\frac{\partial \alpha_{\alpha \beta}}{\partial \chi_{1}^{\gamma}} \frac{\partial \chi_{1}^{F}}{\partial F_{\gamma}}$,

$\gamma_{\alpha \beta \gamma \delta}^{\mathrm{nr}}(0 ; 0,0,0)$

$$
=\sum P_{\alpha \beta \gamma \delta}\left[\frac{1}{6} \frac{\partial \beta_{\alpha \beta \gamma}}{\partial \chi_{l}^{\delta}} \frac{\partial \chi_{1}^{F}}{\partial F_{\delta}}+\frac{1}{8} \frac{\partial \alpha_{\alpha \beta}}{\partial \chi_{2}^{\gamma \delta}} \frac{\partial^{2} \chi_{2}^{F}}{\partial F_{\gamma} \partial F_{\delta}}\right.
$$$$
+\frac{1}{4} \frac{\partial^{2} \alpha_{\alpha \beta}}{\partial \chi_{1}^{\gamma} \partial \chi_{1}^{\delta}} \frac{\partial \chi_{1}^{F}}{\partial F_{\delta}}+\frac{1}{4} \frac{\partial^{2} \mu_{\alpha}}{\partial \chi_{1}^{\beta} \partial \chi_{2}^{\gamma \delta}} \frac{\partial \chi_{1}^{F}}{\partial F_{\beta}} \frac{\partial^{2} \chi_{2}^{F}}{\partial F_{\gamma} \partial F_{\delta}}
$$$$
+\frac{1}{6} \frac{\partial^{3} \mu_{\alpha}}{\partial \chi_{1}^{\beta} \partial \chi_{1}^{\gamma} \partial \chi_{1}^{\delta}} \frac{\partial \chi_{1}^{F}}{\partial F_{\beta}} \frac{\partial \chi_{1}^{F}}{\partial F_{\gamma}} \frac{\partial \chi_{1}^{F}}{\partial F_{\delta}}
$$$$
-\frac{1}{8} \frac{\partial^{3} V}{\partial \chi_{1}^{\alpha} \partial \chi_{1}^{\beta} \partial \chi_{2}^{\gamma \delta}} \frac{\partial \chi_{1}^{F}}{\partial F_{\alpha}} \frac{\partial \chi_{1}^{F}}{\partial F_{\beta}} \frac{\partial^{2} \chi_{2}^{F}}{\partial F_{\gamma} \partial F_{\delta}}
$$$$
\left.-\frac{1}{24} \frac{\partial^{4} V}{\partial \chi_{1}^{\alpha} \partial \chi_{1}^{\beta} \partial \chi_{1}^{\gamma} \partial \chi_{1}^{\delta}} \frac{\partial \chi_{1}^{F}}{\partial F_{\alpha}} \frac{\partial \chi_{1}^{F}}{\partial F_{\beta}} \frac{\partial \chi_{1}^{F}}{\partial F_{\gamma}} \frac{\partial \chi_{1}^{F}}{\partial F_{\delta}}\right] \text {, }
$$

$$
\begin{aligned}
\gamma_{\alpha \beta \gamma \delta}^{\mathrm{nr}}( & -\omega ; \omega, 0,0)_{\alpha \rightarrow \infty} \\
= & \sum P_{\gamma \delta}\left[\frac{\partial \beta_{\alpha \beta \gamma}}{\partial \chi_{1}^{\delta}} \frac{\partial \chi_{1}^{F}}{\partial F_{\delta}}+\frac{1}{2} \frac{\partial \alpha_{\alpha \beta}}{\partial \chi_{2}^{\gamma \delta}} \frac{\partial^{2} \chi_{2}^{F}}{\partial F_{\gamma} \partial F_{\delta}}\right. \\
& \left.+\frac{1}{2} \frac{\partial^{2} \alpha_{\alpha \beta}}{\partial \chi_{1}^{\gamma} \partial \chi_{1}^{\delta}} \frac{\partial \chi_{1}^{F}}{\partial F_{\gamma}} \frac{\partial \chi_{1}^{F}}{\partial F_{\delta}}\right],
\end{aligned}
$$

$$
\begin{aligned}
\gamma_{\alpha \beta \gamma \delta}^{\mathrm{nr}}( & -\omega ; \omega, 0,0)_{\omega \rightarrow \infty} \\
= & \sum P_{\gamma \delta}\left[\frac{\partial \beta_{\alpha \beta \gamma}}{\partial \chi_{1}^{\delta}} \frac{\partial \chi_{1}^{F}}{\partial F_{\delta}}+\frac{1}{2} \frac{\partial \alpha_{\gamma \delta}}{\partial \chi_{2, \mathrm{har}}^{\alpha \beta}}\left(\frac{\partial^{2} \chi_{2, \mathrm{har}}^{F}}{\partial_{\alpha} \partial F_{\beta}}\right)_{\mathrm{har}}\right. \\
& +\frac{1}{2} \frac{\partial^{2} \alpha_{\alpha \beta}}{\partial \chi_{1}^{\gamma} \partial \chi_{1}^{\delta}} \frac{\partial \chi_{1}^{F}}{\partial F_{\gamma}} \frac{\partial \chi_{1}^{F}}{\partial F_{\delta}} \\
& +\frac{\partial^{2} \mu_{\gamma}}{\partial \chi_{1}^{\gamma} \partial \chi_{2, \mathrm{har}}^{\alpha \beta}} \frac{\partial \chi_{1}^{F}}{\partial F_{\delta}}\left(\frac{\partial^{2} \chi_{2, \mathrm{har}}^{F \beta}}{\partial F_{\alpha} \partial F_{\beta}}\right)_{\mathrm{har}} \\
& \left.-\frac{1}{2} \frac{\partial^{3} V}{\partial \chi_{1}^{\gamma} \partial \chi_{1}^{\delta} \partial \chi_{2, \mathrm{har}}^{\alpha \beta}} \frac{\partial \chi_{1}^{F}}{\partial F_{\gamma}} \frac{\partial \chi_{1}^{F}}{\partial F_{\delta}}\left(\frac{\partial^{2} \chi_{2, \mathrm{har}}^{F}}{\partial F_{\alpha} \partial F_{\beta}}\right)_{\mathrm{har}}\right], \\
\gamma_{\alpha \beta \gamma \delta}^{\mathrm{nr}}( & -2 \omega ; \omega, \omega, 0)_{\omega \rightarrow \infty}=\frac{\partial \beta_{\alpha \beta \gamma}}{\partial \chi_{1}^{\delta}} \frac{\partial \chi_{1}^{F}}{\partial F_{\delta}},
\end{aligned}
$$


TABLE I. Structural formula of molecules studied in this paper.

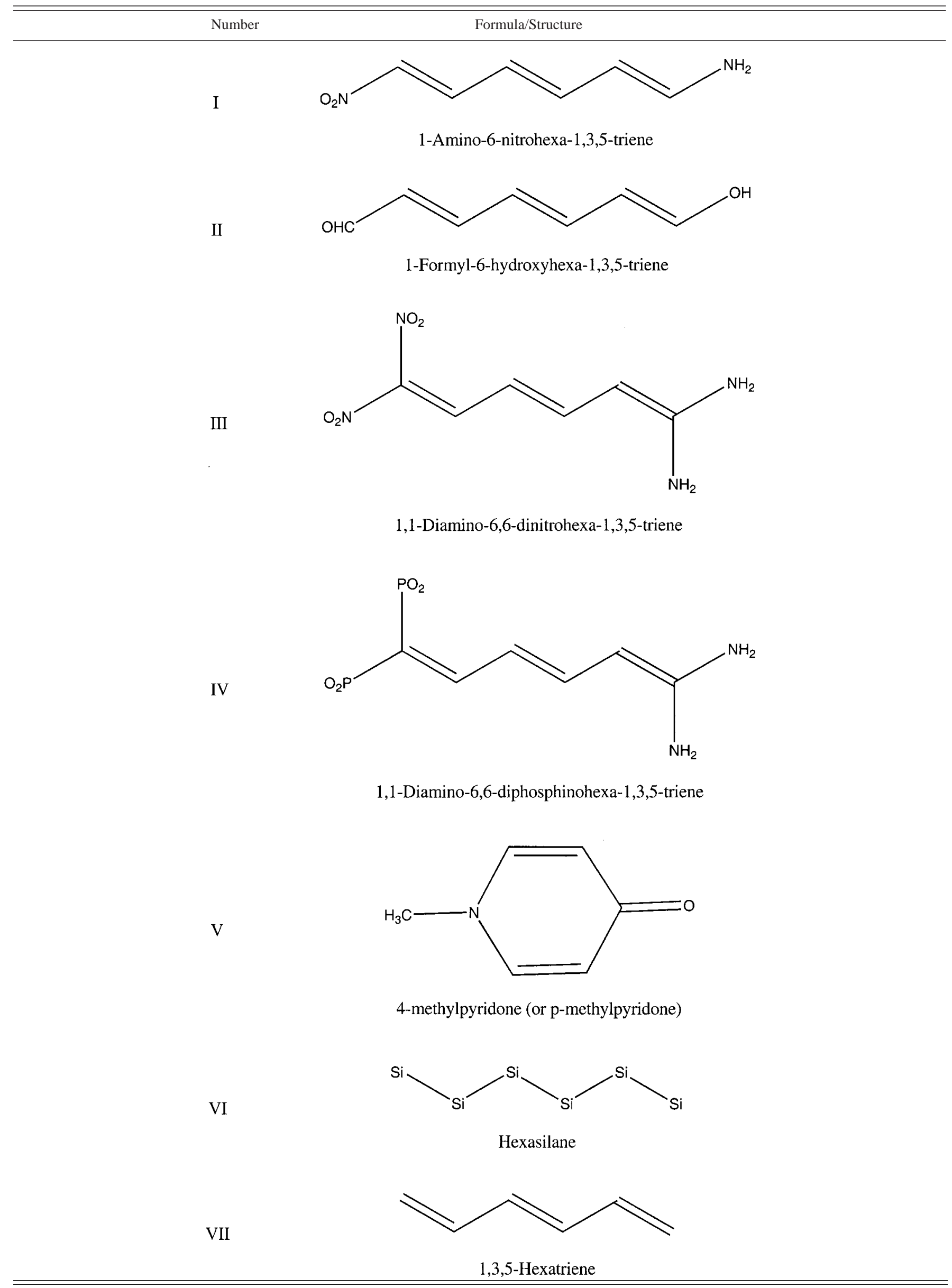


TABLE II. Static polarizabilities $\alpha_{z z}^{e}(0 ; 0), \alpha_{z z}^{\text {zpva }}(0 ; 0), \alpha_{z z}^{\text {nr }}(0 ; 0)$ and $\alpha_{z z}^{\text {c-zpva }}(0 ; 0)$ calculated at the HF/6-31G level in a.u. See Table I for structural formulas of molecules I-VII. The quantity in parentheses is the ratio with respect to $\alpha_{z z}^{e}(0 ; 0)$ multiplied by 100 .

\begin{tabular}{ccccc}
\hline \hline & $\alpha_{z z}^{e}(0 ; 0)$ & $\alpha_{z z}^{\text {zpva }}(0 ; 0)$ & $\alpha_{z z}^{\mathrm{nr}}(0 ; 0)$ & $\alpha_{z z}^{\mathrm{c}-\text { zpva }}(0 ; 0)$ \\
\hline I & $2.39 \times 10^{2}$ & $3.2 \times 10^{0}(1.3)$ & $6.54 \times 10^{1}(27.4)$ & $7.2 \times 10^{0}(3.0)$ \\
II & $2.11 \times 10^{2}$ & $3.46 \times 10^{0}(1.6)$ & $3.84 \times 10^{1}(18.2)$ & $6.68 \times 10^{0}(3.2)$ \\
III & $3.13 \times 10^{2}$ & $1.87 \times 10^{0}(0.6)$ & $3.35 \times 10^{2}(106.8)$ & $-1.51 \times 10^{1}(-4.8)$ \\
IV & $3.22 \times 10^{2}$ & $6.17 \times 10^{0}(1.9)$ & $2.41 \times 10^{2}(74.8)$ & $2 \times 10^{-1}(0.1)$ \\
V & $1.03 \times 10^{2}$ & $2.75 \times 10^{0}(2.7)$ & $1.47 \times 10^{1}(14.3)$ & $7.16 \times 10^{-1}(0.7)$ \\
VI & $2.52 \times 10^{2}$ & $8.66 \times 10^{0}(3.4)$ & $1.08 \times 10^{2}(43.0)$ & $1.06 \times 10^{0}(0.4)$ \\
VII & $1.42 \times 10^{2}$ & $3.70 \times 10^{0}(2.6)$ & $3.13 \times 10^{0}(2.2)$ & $3.08 \times 10^{-1}(0.2)$ \\
\hline \hline
\end{tabular}

$$
\begin{aligned}
& \gamma_{\alpha \beta \delta}^{\mathrm{nr}}(-\omega ; \omega,-\omega, \omega) \\
& \quad=\frac{\partial \alpha_{\alpha \beta}}{\partial \chi_{2, \text { har }}^{\gamma \delta}}\left(\frac{\partial^{2} \chi_{2, \text { har }}^{F}}{\partial F_{\gamma} \partial F_{\delta}}\right)_{\text {har }}+\frac{\partial \alpha_{\alpha \delta}}{\partial \chi_{2, \text { har }}^{\beta \gamma}}\left(\frac{\partial^{2} \chi_{2, \text { har }}^{F}}{\partial F_{\beta} \partial F_{\gamma}}\right)_{\text {har }},
\end{aligned}
$$

where $\Sigma P_{\alpha \beta}$ indicates a sum over both permutations of the indices $\alpha$ and $\beta$. Here $\chi_{1}^{F}$, for example, is obtained by replacing $Q_{i}$ with $Q_{i}^{F}$ in Eq. (2). The number of FICs necessary for any calculation depends upon the property and which elements of the tensor are desired. For instance, for $\gamma_{\alpha \beta \gamma \delta}^{\mathrm{nr}}(-2 \omega ; \omega, \omega, 0)_{\omega \rightarrow \infty}$ only $\chi_{1}^{\delta}$ is required [cf. Eq. (14)]; whereas for $\gamma_{\alpha \beta \gamma \delta}^{\mathrm{nr}}(-\omega ; \omega, 0,0)_{\omega \rightarrow \infty}$ one needs $\chi_{1}^{\gamma}, \chi_{1}^{\delta}, \chi_{2, \text { har }}^{\alpha \beta}$ (or $\chi_{2}^{\alpha \beta}$ ) [cf. Eq. (13)].

The first-order static ZPVA contribution to any property is given by the sum of a first-order term in electrical anharmonicity plus a first-order term in mechanical anharmonicity. ${ }^{31}$ Although FICs cannot be employed to simplify the former, the mechanical anharmonicity term can be expressed as a function of the harmonic coordinates, which yields the total first-order static ZPVA correction: ${ }^{24,32}$

$$
\begin{aligned}
P^{\mathrm{zpva}}=[P]^{1,0}+[P]^{0,1}= & -\frac{\hbar^{3 N-6}}{4} \sum_{i}\left(\frac{1}{\omega_{i}} \frac{\partial^{n+2} V}{\partial Q_{i}^{2} \partial F^{n}}\right) \\
& -\frac{\partial E^{z p}}{\partial \chi_{n, \text { har }}}\left(\frac{\partial^{n} \chi_{n, \text { har }}^{F}}{\partial F^{n}}\right)_{\text {har }},
\end{aligned}
$$

where

$$
E^{z p}=\hbar / 2 \sum_{i}^{3 N-6} \omega_{i}
$$

$\omega_{i}$ is a harmonic vibrational frequency, and $n$ is 1 for the dipole moment, 2 for the linear polarizability, 3 for the first hyperpolarizability and 4 for the second hyperpolarizability. For convenience, the designation of the components of the electric field, $F$, has been suppressed. The C-ZPVA contribution corresponding to Eq. (16) can be expressed as a sum of the same type of square bracket terms as the NR contribution except that, for C-ZPVA, these terms are of the next (nonvanishing) higher-order of perturbation theory. For instance, the NR contribution to the static $\gamma^{5,23}$ is-

$$
\begin{aligned}
\gamma_{\alpha \beta \gamma \delta}^{\mathrm{nr}}(0 ; 0,0,0)= & {\left[\alpha^{2}\right]_{\omega=0}^{0}+[\mu \beta]_{\omega=0}^{0}+\left[\mu^{2} \alpha\right]_{\omega=0}^{\mathrm{I}} } \\
& +\left[\mu^{4}\right]_{\omega=0}^{\mathrm{II}},
\end{aligned}
$$

whereas the C-ZPVA contribution is given by

$$
\begin{aligned}
\gamma_{\alpha \beta \gamma \delta}^{\mathrm{c}-\mathrm{pvva}}(0 ; 0,0,0)= & {\left[\alpha^{2}\right]_{\omega=0}^{\mathrm{II}}+[\mu \beta]_{\omega=0}^{\mathrm{II}}+\left[\mu^{2} \alpha\right]_{\omega=0}^{\mathrm{III}} } \\
& +\left[\mu^{4}\right]_{\omega=0}^{\mathrm{IV}} .
\end{aligned}
$$

Although all even-order terms in the total ZPVA property expression vanish, there are contributions for all odd orders of perturbation theory. Each time the order is increased by two so is the order of each square bracket term in the higherorder analogue of Eq. (19).

The NR formulas given in Eqs. (8)-(15) contain derivatives of the potential energy up to fourth order either in the field, or the vibrational coordinates, or a combination of the two. Since the C-ZPVA contributions are two orders of perturbation theory higher, it is not surprising that the corresponding equations will contain sixth derivatives of the potential energy. Numerical computation of the sixth derivatives with respect to nuclear displacements is extremely time-consuming and subject to large relative errors (they cannot be computed analytically using standard quantum chemistry program packages). Fortunately, under the infinite optical frequency approximation, the C-ZPVA contributions can be determined alternatively by means of the KLB finite field method. ${ }^{23}$ The KLB technique is exactly analogous to the BHK method for the NR contribution ex-

TABLE III. First hyperpolarizabilities $\beta_{z z z}^{e}(0 ; 0,0), \beta_{z z z}^{\text {zpva }}(0 ; 0,0), \beta_{z z z}^{\text {nr }}(0 ; 0,0), \beta_{z z z}^{\mathrm{c}-z p v a}(0 ; 0,0), \beta_{z z z}^{\mathrm{nr}}(-\omega ; \omega, 0)_{\omega \rightarrow \infty}$, and $\beta_{z z z}^{\mathrm{c}-z p v a}(-\omega ; \omega, 0){ }_{\omega \rightarrow \infty}$ calculated at the $\mathrm{HF} / 6-31 \mathrm{G}$ level in a.u. See Table I for structural formula of molecules I-V. The quantity in parentheses is the ratio with respect to $\beta_{z z z}^{e}(0 ; 0,0)$ multiplied by 100.

\begin{tabular}{ccccccc}
\hline \hline & $\beta_{z z z}^{e}(0 ; 0,0)$ & $\beta_{z z z}^{\text {zpva }}(0 ; 0,0)$ & $\beta_{z z z}^{\text {nr }}(0 ; 0,0)$ & $\beta_{z z z}^{\mathrm{c}-z p v a}(0 ; 0,0)$ & $\beta_{z z z}^{\text {nr }}(-\omega ; \omega, 0)_{\omega \rightarrow \infty}$ & $\beta_{z z z}^{\mathrm{c}-\text { zpva }}(-\omega ; \omega, 0)_{\omega \rightarrow \infty}$ \\
\hline I & $4.28 \times 10^{3}$ & $-2.4 \times 10^{2}(-5.6)$ & $1.30 \times 10^{4}(303.7)$ & $-4.6 \times 10^{1}(-1.1)$ & $2.99 \times 10^{3}(69.9)$ & $1.5 \times 10^{2}(3.5)$ \\
II & $1.79 \times 10^{3}$ & $-8.78 \times 10^{1}(-4.9)$ & $3.96 \times 10^{3}(220.9)$ & $-1.1 \times 10^{3}(-59.1)$ & $1.11 \times 10^{3}(62.1)$ & $8.32 \times 10^{1}(4.6)$ \\
III & $2.20 \times 10^{2}$ & $-5.09 \times 10^{1}(-23.2)$ & $5.20 \times 10^{2}(236.9)$ & $2.3 \times 10^{3}(1051.0)$ & $3.25 \times 10^{3}(1481.7)$ & $1.81 \times 10^{2}(82.5)$ \\
IV & $-2.88 \times 10^{3}$ & $4.7 \times 10^{2}(-16.2)$ & $-2.79 \times 10^{4}(968.6)$ & $-2.9 \times 10^{2}(10.2)$ & $-2.69 \times 10^{3}(93.4)$ & $8.48 \times 10^{2}(-29.5)$ \\
V & $-3.31 \times 10^{2}$ & $-1.61 \times 10^{1}(4.9)$ & $2.44 \times 10^{2}(-73.6)$ & $9.9 \times 10^{0}(-3.0)$ & $6.88 \times 10^{1}(-20.8)$ & $-1.17 \times 10^{-1}(0.04)$ \\
\hline \hline
\end{tabular}




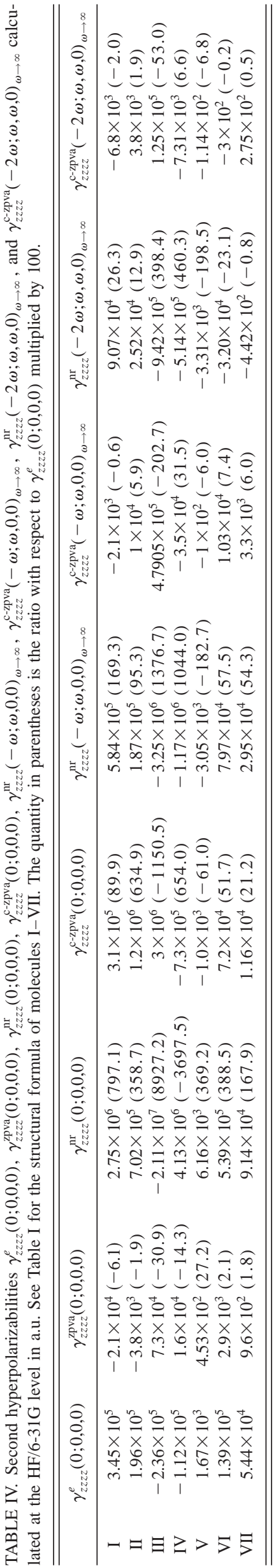

cept that $P^{e}$ is replaced by $P^{\text {zpva }}$. In this instance one carries out a power series expansion of the static field-dependent ZPVA correction to the property value taking into account the relaxation of equilibrium geometry due to the field:

$$
\begin{aligned}
& \Delta \mu_{\alpha}^{\text {zpva }}=a_{\alpha \beta}^{1, \text { zpva }} F_{\beta}+\frac{b_{\alpha \beta \gamma}^{1, \text { zpva }}}{2} F_{\beta} F_{\gamma}+\frac{g_{\alpha \beta \gamma \delta}^{1, \text { zpva }}}{6} F_{\beta} F_{\gamma} F_{\delta}+\cdots, \\
& \Delta \alpha_{\alpha \beta}^{\text {zpva }}=b_{\alpha \beta \gamma}^{2, \text { zpva }} F_{\gamma}+\frac{g_{\alpha \beta \gamma \delta}^{2, \text { zpva }}}{2} F_{\gamma} F_{\delta}+\cdots \\
& \Delta \beta_{\alpha \beta \gamma}^{\text {zpva }}=g_{\alpha \beta \gamma \delta}^{3, \text { zpva }} F_{\delta}+\cdots
\end{aligned}
$$

The coefficients in Eqs. (20)-(22) yield the C-ZPVA static and infinite optical frequency (hyper)polarizabilities:

$$
\begin{aligned}
& a_{\alpha \beta}^{1, \text { zpva }}=\alpha_{\alpha \beta}^{\text {zpva }}(0 ; 0)+\alpha_{\alpha \beta}^{\mathrm{c}-\text { zpva }}(0 ; 0), \\
& b_{\alpha \beta \gamma}^{1, \text { zpva }}=\beta_{\alpha \beta \gamma}^{\text {zpva }}(0 ; 0,0)+\beta_{\alpha \beta \gamma}^{\mathrm{c}-\mathrm{zpva}}(0 ; 0,0), \\
& g_{\alpha \beta \gamma \delta}^{1, \mathrm{zpva}}=\gamma_{\alpha \beta \gamma \delta}^{\mathrm{zpva}}(0 ; 0,0,0)+\gamma_{\alpha \beta \gamma \delta}^{\mathrm{c}-\mathrm{zpva}}(0 ; 0,0,0), \\
& b_{\alpha \beta \gamma}^{2, \mathrm{zpva}}=\beta_{\alpha \beta \gamma}^{\text {zpva }}(0 ; 0,0)+\beta_{\alpha \beta \gamma}^{\mathrm{c}-\mathrm{zpva}}(-\omega ; \omega, 0)_{\omega \rightarrow \infty}, \\
& g_{\alpha \beta \gamma \delta}^{2, \mathrm{zpva}}=\gamma_{\alpha \beta \gamma \delta}^{\mathrm{zpva}}(0 ; 0,0,0)+\gamma_{\alpha \beta \gamma \delta}^{\mathrm{c}-\mathrm{zpva}}(-\omega ; \omega, 0,0)_{\omega \rightarrow \infty}, \\
& g_{\alpha \beta \gamma \delta}^{3, \mathrm{zpva}}=\gamma_{\alpha \beta \gamma \delta}^{\mathrm{zpva}}(0 ; 0,0,0)+\gamma_{\alpha \beta \gamma \delta}^{\mathrm{c}-\mathrm{zpva}}(-2 \omega ; \omega, \omega, 0)_{\omega \rightarrow \infty} .
\end{aligned}
$$

\section{COMPUTATIONAL CONSIDERATIONS}

In order to see whether the conclusions obtained in previous investigations of the typical push-pull polyene series $\mathrm{NH}_{2}-(\mathrm{CH}=\mathrm{CH})_{n}-\mathrm{NO}_{2}$ (I) ${ }^{22,24}$ can be generalized, we added the molecules 1-formyl-6-hydroxyhexa-1,3,5-triene (II), 1,1-diamino-6,6-dinitrohexa-1,3,5-triene (III), 1,1diamino-6,6-diphosphinohexa-1,3,5-triene (IV), 4-methylpyridone (V), hexasilane (VI) and 1,3,5-hexatriene (VII) (see Table I). This set of medium-size organic molecules contains representatives of three different types of compound in terms of polarity and valence bond-charge transfer (VB-CT) characteristics, as classified according to their electronic (hyper)polarizabilities. ${ }^{33}$ I and II are polar with a dominant VB ground state; III and IV are polar with a ground state that has mixed VB-CT character; $\mathrm{V}$ is polar with a dominant $\mathrm{CT}$ ground state; and VI and VII are nonpolar.

All dynamic vibrational properties were calculated in the infinite optical frequency $(\omega \rightarrow \infty)$ limit, which is equivalent to, assuming that $\left(\omega_{i} / \omega\right)^{2}$ is negligible compared to unity for each harmonic vibrational frequency. The NR and ZPVA contributions were computed using the analytic FIC expressions [Eqs. (8)-(14) and (16)], whereas the C-ZPVA contribution was obtained using the finite field KLB procedure. ${ }^{23}$

Many of the lower-order derivatives required for our treatment were obtained analytically using the GAUSSIAN 98 suite of programs. ${ }^{34}$ These include $a_{20}, a_{01}, a_{11}, a_{02}, a_{12}$, and $a_{03}$ at the HF level and all but the last two of this list at the MP2 level. All vibrational derivatives are computed with respect to atomic Cartesian coordinates. In order to evaluate $[P]^{1,0}$ we need the complete Hessian (i.e., the matrix corresponding to $a_{20}$ in Cartesian coordinates), as a function of an applied static field [see Eq. (16)]. The latter may be deter- 
TABLE V. Static polarizabilities $\alpha_{z z}^{e}(0 ; 0), \alpha_{z z}^{\text {zpva }}(0 ; 0), \alpha_{z z}^{\mathrm{nr}}(0 ; 0)$, and $\alpha_{z z}^{\mathrm{c}-z \mathrm{pva}}(0 ; 0)$ calculated at the MP2/6$31 \mathrm{G}$ level in a.u. See Table I for structural formula of molecules I-VII. The quantity in parentheses is the ratio with respect to $\alpha_{z z}^{e}(0 ; 0)$ multiplied by 100 .

\begin{tabular}{ccccc}
\hline \hline & $\alpha_{z z}^{e}(0 ; 0)$ & $\alpha_{z z}^{\text {zpva }}(0 ; 0)$ & $\alpha_{z z}^{\text {nr }}(0 ; 0)$ & $\alpha_{z z}^{\text {c-zpva }}(0 ; 0)$ \\
\hline I & $2.72 \times 10^{2}$ & $-2.33 \times 10^{0}(-0.9)$ & $3.53 \times 10^{1}(13.0)$ & $4.30 \times 10^{1}(15.8)$ \\
II & $2.09 \times 10^{2}$ & $-2.89 \times 10^{-1}(-0.1)$ & $2.62 \times 10^{1}(12.5)$ & $2.85 \times 10^{0}(1.4)$ \\
III & $3.75 \times 10^{2}$ & $6.85 \times 10^{0}(1.8)$ & $2.79 \times 10^{2}(74.6)$ & $-1.92 \times 10^{1}(-5.1)$ \\
IV & $4.70 \times 10^{2}$ & $5.42 \times 10^{0}(1.2)$ & $4.78 \times 10^{2}(101.6)$ & $\cdots$ \\
V & $1.09 \times 10^{2}$ & $3.33 \times 10^{0}(3.0)$ & $9.84 \times 10^{0}(9.0)$ & $-6.8 \times 10^{-3}(-0.01)$ \\
VI & $2.66 \times 10^{2}$ & $8.14 \times 10^{0}(3.1)$ & $1.08 \times 10^{2}(40.6)$ & $-9.84 \times 10^{-1}(-0.4)$ \\
VII & $1.25 \times 10^{2}$ & $9.1 \times 10^{-1}(0.7)$ & $1.82 \times 10^{0}(1.5)$ & $1.64 \times 10^{-1}(0.1)$ \\
\hline \hline
\end{tabular}

mined analytically from GAUSSIAN 98 either at the HartreeFock (HF) or MP2 level, although the A.10 revision must be used for the correlated treatment.

At the HF level, a single numerical differentiation of the analytical $a_{20}, a_{11}, a_{12}$, and $a_{03}$ with respect to the appropriate FICs yields the necessary $a_{30}, a_{21}, a_{22}$, and $a_{13}$, respectively. The required $a_{40}$ and $a_{31}$ were computed by double numerical differentiation of $a_{20}$ and $a_{11}$. Finally, $a_{04}$ was calculated by double numerical differentiation of $a_{02}$ with respect to an electric field. At the MP2 level, a single numerical differentiation of the analytical $a_{20}$ and $a_{11}$ with respect to the FICs gives $a_{30}$, and $a_{21}$, respectively, whereas double numerical differentiation of $a_{20}, a_{11}$, and $a_{02}$ yields $a_{40}, a_{31}$, and $a_{22}$. Single and double differentiation of $a_{02}$ with respect to an electric field leads to the derivatives $a_{03}$ and $a_{04}$, respectively, while single and double differentiation of $a_{11}$ gives $a_{12}$ and $a_{13}$.

The magnitude of the displacement used for the numerical derivatives with respect to vibrational coordinates was 0.04 a.u. and the stability of the derivatives was checked by repeating the calculation with the magnitude of the displacement doubled. In connection with Eq. (16) one must be careful to stay within the window of field values where the fourth derivative of the Hessian with respect to the field is stable. This was accomplished by carrying out calculations for fields of $\pm 0.0004, \pm 0.0008, \pm 0.0016, \pm 0.0032, \pm 0.0064$, \pm 0.0128 , and \pm 0.0256 a.u. Then, for each molecule, a Romberg table was constructed ${ }^{1,35}$ and the smallest magnitude field that produced a stable derivative was selected. This systematic procedure allowed us to control the magnitude of the error of the numerical derivatives with respect to the electric field.

ZPVA corrections were calculated at the field-free, and several field-dependent equilibrium geometries in order to obtain the C-ZPVA contributions. The field-dependent geom- etry optimizations were carried out with the Eckart conditions strictly enforced. ${ }^{27}$ Finally, the coefficients in Eqs. (20)-(22) were obtained by means of the Romberg technique $^{35}$ using fields of $\pm 0.0004, \pm 0.0008, \pm 0.0016$, \pm 0.0032 , and \pm 0.0064 a.u. (for molecule $\mathrm{I}$ it was necessary to add fields of \pm 0.0002 a.u.). The magnitude of the numerical errors is of the same order as the last figure given in the data presented in the Tables II-VIII.

The 6-31G basis set ${ }^{36}$ was employed in these calculations. A number of investigations have shown ${ }^{1,37,38}$ that, in the case of quasilinear molecules, this basis gives semiquantitative accuracy for the longitudinal component of the (hyper)polarizability tensor, which is the most important component. Using the $6-31 \mathrm{G}$ basis, we can handle molecules such as 1,1-diamino-6,6-diphosphinohexa-1,3,5-triene, which contains 12 second row and 2 third row atoms. However, an MP2 treatment of the C-ZPVA contribution for molecules III and IV still proved to be beyond the reach of our computational facilities.

\section{RESULTS}

Tables II-IV and V-VII summarize the HF and MP2 results we have obtained for the longitudinal component of $P^{e}, P^{\mathrm{zpva}}, P^{\mathrm{nr}}$ and $P^{\mathrm{c}-\mathrm{zpva}}$ in molecules I-VII. For molecule I, in particular, the HF results were taken from Ref. 24. Let us begin by examining the effect of electron correlation on $\alpha(0 ; 0)$ in Tables II and V. For $\alpha^{e}(0 ; 0)$ the correlation effect, in general, is relatively small. It is somewhat larger for molecule IV than the others but the ordering is preserved. As anticipated, the differences between HF and MP2 are larger for $\alpha^{\mathrm{nr}}(0 ; 0)$ than $\alpha^{e}(0 ; 0)$. However, except for a reversal in order between molecules III and IV, the trends are the same in either case, and the relative magnitude of the NR versus electronic polarizability is similar. When it comes to the

TABLE VI. First hyperpolarizabilities $\beta_{z z z}^{e}(0 ; 0,0), \beta_{z z z}^{\text {zpva }}(0 ; 0,0), \beta_{z z z}^{\mathrm{nr}}(0 ; 0,0), \beta_{z z z}^{\mathrm{c}-\text { pva }}(0 ; 0,0), \beta_{z z z}^{\mathrm{nr}}(-\omega ; \omega, 0)_{\omega \rightarrow \infty}$, and $\beta_{z z z}^{\mathrm{c}-\text { ppa }}(-\omega ; \omega, 0)_{\omega \rightarrow \infty}$ calculated at the MP2/6-31G level in a.u. See Table I for structural formula of molecules I-V. The quantity in parentheses is the ratio with respect to $\beta_{z z z}^{e}(0 ; 0,0)$ multiplied by 100 .

\begin{tabular}{ccccccc}
\hline \hline & $\beta_{z z z}^{e}(0 ; 0,0)$ & $\beta_{z z z}^{\text {zpva }}(0 ; 0,0)$ & $\beta_{z z z}^{\text {nr }}(0 ; 0,0)$ & $\beta_{z z z}^{\text {c-zpva }}(0 ; 0,0)$ & $\beta_{z z z}^{\text {nr }}(-\omega ; \omega, 0)_{\omega \rightarrow \infty}$ & $\beta_{z z z}^{\mathrm{c}-\text { zpva }}(-\omega ; \omega, 0)_{\omega \rightarrow \infty}$ \\
\hline I & $1.24 \times 10^{4}$ & $-3.4 \times 10^{2}(-2.7)$ & $8.90 \times 10^{3}(71.5)$ & $-3.6 \times 10^{4}(-287.2)$ & $2.68 \times 10^{3}(21.5)$ & $1.23 \times 10^{3}(9.9)$ \\
II & $5.05 \times 10^{3}$ & $6.3 \times 10^{1}(1.2)$ & $2.90 \times 10^{3}(57.5)$ & $-7.03 \times 10^{2}(-13.9)$ & $9.49 \times 10^{2}(18.8)$ & $7.00 \times 10^{1}(1.4)$ \\
III & $1.32 \times 10^{4}$ & $4.2 \times 10^{1}(0.3)$ & $6.56 \times 10^{4}(496.8)$ & $-1.70 \times 10^{4}(-129.1)$ & $1.26 \times 10^{4}(95.6)$ & $-1.03 \times 10^{2}(-0.8)$ \\
IV & $2.59 \times 10^{3}$ & $9.67 \times 10^{2}(37.3)$ & $1.5 \times 10^{3}(56.4)$ & $\cdots$ & $5.18 \times 10^{3}(199.5)$ & $\cdots$ \\
V & $1.69 \times 10^{1}$ & $3.8 \times 10^{0}(22.8)$ & $8.85 \times 10^{1}(523.5)$ & $1.30 \times 10^{2}(768.9)$ & $8.29 \times 10^{1}(490.7)$ & $6.24 \times 10^{0}(36.9)$ \\
\hline \hline
\end{tabular}




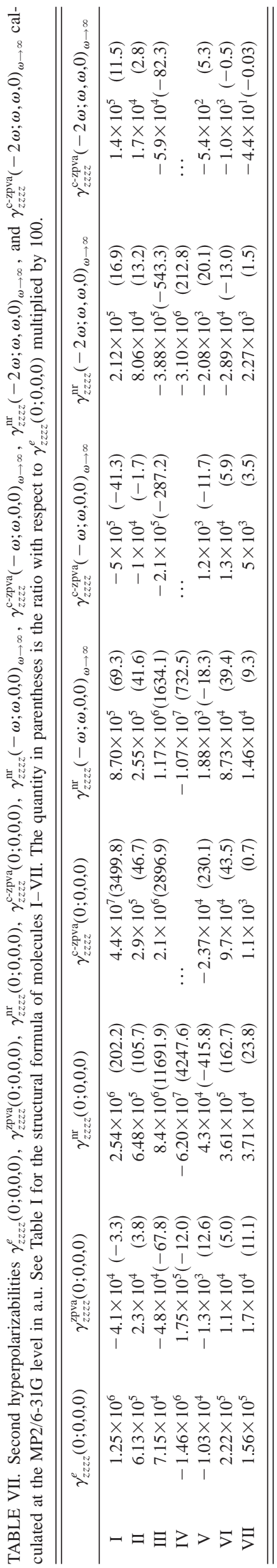

properties that depend upon anharmonicity, i.e., $\alpha^{\mathrm{zpva}}(0 ; 0)$ and $\alpha^{\text {c-zpva }}(0 ; 0)$, the correlation effect is more serious. For example, at the HF level the C-ZPVA value for the static polarizability of molecule I is about ten times smaller than the NR value whereas, at the MP2 level, the C-ZPVA value is the larger of the two. Thus, the HF results lead to an erroneous conclusion about the initial convergence of series $\mathrm{B}$ in this case. Since the vibrational contributions are considerably smaller than $\alpha^{e}$, whether correlation is included or not, this discrepancy may not be too significant for the static polarizability.

For hyperpolarizabilities, on the other hand, the situation is different. HF and MP2 first hyperpolarizabilities are reported in Tables III and VI. Using either table we see that the NR contribution to the static $\beta$ and to the dc-Pockels effect $(\beta(-\omega ; \omega, 0)$; dc-P $)$ may be up to an order of magnitude larger than the static $\beta^{e}$. The same is true of the C-ZPVA contribution although, in general (see later), it is the smaller of the two. For both NR and C-ZPVA there is a strong (though not universal) tendency for the dc-P $\beta$ to be smaller than the static value. In terms of both magnitude and sign there are dramatic changes between the HF and MP2 results. Taking molecule I as the example, once again, the relative importance of the NR and C-ZPVA contributions to the static $\beta$ is reversed when correlation is taken into account; and this is also true for molecules III and V. It has been observed ${ }^{22}$ that the magnitude of the ratio $\beta^{n r} / \beta^{e}(0 ; 0,0)$ is significantly reduced by electron correlation in the cases previously studied. We find that this happens for only about half the molecules considered here.

All of the behaviors described for $\beta$ pertain as well to $\gamma$ except that the relative importance of the vibrational properties is further enhanced. Thus, by examining Tables IV and VII we see that the NR and C-ZPVA (hyper)polarizabilities may be up to two orders of magnitude larger than the static $\gamma^{e}$. The magnitude of the vibrational contribution depends upon the NLO process, and there is a strong tendency for it to increase as the number of static fields increases. Thus, the static value generally exceeds that of the optical Kerr effect (OKE), $\gamma(-\omega ; \omega, 0,0)$, which in turn is greater than dc-second harmonic generation (dc-SHG), $\gamma(-2 \omega ; \omega, \omega, 0)$. Again, the effect of electron correlation is very large leading to numerous reversals in the relative importance of the NR and C-ZPVA contributions, but no systematic increase or decrease in the magnitude of the ratio with respect to the static $\gamma^{e}$ is found.

From the tables presented here it is clear that we should focus on the MP2 results in analyzing the initial convergence of the (A) and (B) series for (hyper)polarizabilities. It turns out that the same general conclusions would also emerge from the HF calculations even though the specifics regarding individual molecules would change.

As far as series (A) is concerned, the ZPVA contribution to the static polarizability is always less than $3.2 \%$ of the corresponding electronic term. On the other hand, for the static first hyperpolarizability the ratio $\left|P^{\text {zpva }} / P^{e}\right|$ varies from .003 (III) up to .373 (IV) while, for the second hyperpolarizability, it ranges between .033 (I) and .678 (III). It is clear that the ZPVA correction is not systematically negligible, 
TABLE VIII. Electrical $\left([P]^{1,0}\right)$ and mechanical $\left([P]^{0,1}\right)$ anharmonicity contributions to $\alpha_{z z}^{\text {zpva }}(0 ; 0), \beta_{z z z}^{\text {zpva }}(0 ; 0,0)$, and $\gamma_{z z z}^{\text {zpva }}(0 ; 0,0,0)$ calculated at the MP2/6-31G level in a.u. See Table I for structural formula of molecules I-VII. The quantity in parentheses is the ratio with respect to the corresponding $P^{\text {zpva }}$ multiplied by 100 .

\begin{tabular}{cccccrr}
\hline \hline & {$\left[\alpha^{\text {zpa }}(0 ; 0)\right]^{1,0}$} & {$\left[\alpha^{\text {zpva }}(0 ; 0)\right]^{0,1}$} & {$\left[\beta^{\text {zpva }}(0 ; 0,0)\right]^{1,0}$} & {$\left[\beta^{\text {zpva }}(0 ; 0,0)\right]^{0,1}$} & {$\left[\gamma^{\text {zpva }}(0 ; 0,0,0)\right]^{1,0}$} & {$\left[\gamma^{\text {zpva }}(0 ; 0,0,0)\right]^{0,1}$} \\
\hline I & $4.22 \times 10^{0}(-181.0)$ & $-6.55 \times 10^{0}(281.0)$ & $1.3 \times 10^{2}(-37.6)$ & $-4.7 \times 10^{2}(137.6)$ & $-4.5 \times 10^{4}(110.0)$ & $4.1 \times 10^{3}(-10.0)$ \\
II & $1.44 \times 10^{0}(-500.0)$ & $-1.73 \times 10^{0}(600.0)$ & $1.7 \times 10^{2}(273.7)$ & $-1.1 \times 10^{2}(-173.7)$ & $2.8 \times 10^{4}(120.8)$ & $-4.8 \times 10^{3}(-20.8)$ \\
III & $9.83 \times 10^{0}(143.5)$ & $-2.98 \times 10^{0}(-43.5)$ & $-2.6 \times 10^{2}(-608.9)$ & $3.0 \times 10^{2}(708.9)$ & $-1.3 \times 10^{5}(275.0)$ & $8.5 \times 10^{4}(-175.0)$ \\
IV & $4.81 \times 10^{0}(88.8)$ & $6.04 \times 10^{-1}(11.2)$ & $-1.52 \times 10^{1}(-1.6)$ & $9.82 \times 10^{2}(101.6)$ & $1.81 \times 10^{5}(103.1)$ & $-5.41 \times 10^{3}(-3.1)$ \\
V & $3.15 \times 10^{0}(94.7)$ & $1.75 \times 10^{-1}(5.3)$ & $-7.8 \times 10^{0}(-201.7)$ & $1.2 \times 10^{1}(301.7)$ & $-9.7 \times 10^{2}(74.7)$ & $-2.8 \times 10^{2}(21.5)$ \\
VI & $9.89 \times 10^{0}(121.4)$ & $-1.75 \times 10^{0}(-21.4)$ & $\ldots$ & $\ldots$ & $1.4 \times 10^{4}(122.9)$ & $-2.5 \times 10^{3}(-22.9)$ \\
VII & $8.6 \times 10^{-1}(94.2)$ & $5.3 \times 10^{-2}(5.8)$ & $\ldots$ & $\cdots$ & $1.6 \times 10^{4}(90.6)$ & $1.6 \times 10^{3}(9.4)$ \\
\hline \hline
\end{tabular}

though it is less than $13 \%$ of the corresponding static electronic property in most cases. In all instances $P^{\text {zpva }}$ is smaller than $P^{e}$ which means that series $(\mathrm{A})$ is always initially convergent. Table VIII presents the breakdown of $P^{\text {zpva }}$ into electrical and mechanical anharmonicity contributions. For $\alpha^{\text {zpva }}$ and $\gamma^{\text {zpva }}$ the electrical anharmonicity is often, but not always, dominant. On the other hand, for $\beta^{\text {zpva }}$ the mechanical anharmonicity term is larger for four of the five molecules.

Evidently, the NR (hyper)polarizability, which is the first term of series (B), should be systematically computed. In agreement with previous studies ${ }^{1,13}$ this contribution to the vibrational (hyper)polarizability is often substantially larger than, or is comparable to, its static electronic counterpart for the molecules considered here. Note that this applies to dynamic as well as static processes. The initial convergence of series (B) may be considered satisfactory if $P^{\text {c-zpva }}$ is substantially smaller than $P^{\mathrm{nr}}$ for the same property. For $d y$ namic hyperpolarizabilities $\left|P^{\mathrm{c}-z p v a} / P^{\mathrm{nr}}\right|$ is always less than 0.66 ; usually this ratio is much smaller. Thus, the initial convergence criterion is met although the situation is borderline in a couple of instances. It is nonetheless important to note that the dynamic C-ZPVA hyperpolarizabilities are typically comparable in magnitude to the corresponding static $P^{\text {zpva }}$ and, consequently, may not be negligible with respect to the corresponding static $P^{e}$. For example, $\left|P^{\mathrm{c}-\mathrm{zpva}} / P^{e}(0 ; 0,0,0)\right|$ is 0.37 for dc-P of molecule $\mathrm{V}$; 0.41 for $\mathrm{OKE}$ of molecule $\mathrm{I}$; and 0.83 for dc-SHG of molecule III.

For static (hyper)polarizabilities the initial convergence of the (B) series is not completely satisfactory. The static linear polarizability meets the criterion quite adequately in almost all instances. That is to say, the ratio $\left|\alpha^{\mathrm{c}-\mathrm{zpva}} / \alpha^{\mathrm{nr}}\right|$ is less than 0.11 except for molecule I which has a ratio of 1.2; in this molecule, however, $\alpha^{\mathrm{c}-\mathrm{zpva}}$ is only $15.8 \%$ of $\alpha^{e}$. For the static first hyperpolarizability there are two cases where the (B) series is not initially convergent, namely $\left|\beta^{\mathrm{c}-z p v a} / \beta^{\mathrm{nr}}\right|=4.0$ for molecule I and 1.5 for molecule $\mathrm{V}$. In addition, the magnitude of $\beta^{\mathrm{c}-z p v a}$ is greater than $\beta^{e}$ for both of these molecules. A similar circumstance occurs for the static second hyperpolarizability of molecule I, i.e., $\left|\gamma^{\mathrm{c}-z p v a} / \gamma^{\mathrm{nr}}\right|=17.32$ and $\gamma^{\mathrm{c}-\mathrm{zpva}}>\gamma^{e}$. Although $\left|P^{\mathrm{c}-\mathrm{zpva}} / P^{\mathrm{nr}}\right|$ is smaller than 0.56 in all other instances, the above results demonstrate that: $(1)$ it is important to calculate $P^{\mathrm{c}-z p v a}$, particularly for static (hyper)polarizabilities, even when $P^{\text {zpva }}$ is relatively small, and (2) the initial convergence behavior of the perturbation treatment of electrical/mechanical anharmonicity for ordinary molecules remains an open issue. The fact that the static $\beta^{\mathrm{c}-z p v a}$ and $\gamma^{\mathrm{c} \text {-zpva }}$ contributions are the most problematic as far as initial convergence is concerned suggests that the $\left[\mu^{3}\right]^{\mathrm{III}}$ and $\left[\mu^{4}\right]^{\mathrm{IV}}$ terms are primarily responsible. This follows from the fact that these terms appear in the expressions for the static properties but not the (infinite optical frequency) dynamic processes. We have shown elsewhere that their magnitude decreases rapidly when one of the optical frequencies is larger than 0.02 a.u. $\left(4389 \mathrm{~cm}^{-1}\right){ }^{18}$

It is of interest to anatomize the static contributions to the hyperpolarizabilities into harmonic and anharmonic terms. In Table IX we provide such a breakdown of $\beta_{z z z}^{\mathrm{nr}}(0 ; 0,0), \beta_{z z z}^{\mathrm{c}-z \mathrm{pva}}(0 ; 0,0)$ and $\gamma_{z z z z}^{\mathrm{nr}}(0 ; 0,0,0)$ at the MP2 level. For $\beta^{\mathrm{nr}}(0 ; 0,0)$, the $\left[\mu^{3}\right]^{\mathrm{I}}$ term is negligible in molecules I and II, but must be taken into account in molecules III-V. For $\gamma^{\mathrm{nr}}(0 ; 0,0,0)$ the $\left[\mu^{4}\right]^{\mathrm{II}}$ term is less than $13 \%$ of the total in molecules I-III and VII, but greater than $\sim 40 \%$ in the other three. Using a cutoff of $20 \%$, the $\left[\mu^{2} \alpha\right]^{\mathrm{I}}$ term can be neglected only for molecules II, IV, and VII. Thus, in

TABLE IX. Breakdown of MP2/6-31G $\beta_{z z}^{\mathrm{nr}}(0 ; 0,0), \beta_{z z z}^{\mathrm{c}-z p \mathrm{a}}(0 ; 0,0)$, and $\gamma_{z z z z}^{\mathrm{nr}}(0 ; 0,0,0)$ square bracket terms. See Table I for structural formula of molecules I-VII. All quantities are in a.u.

\begin{tabular}{|c|c|c|c|c|c|c|c|c|}
\hline & \multicolumn{2}{|c|}{$\beta_{z z z}^{\mathrm{nn}}(0 ; 0,0)$} & \multicolumn{2}{|c|}{$\beta_{z z z}^{\mathrm{c}-\mathrm{zpva}}(0 ; 0,0)$} & \multicolumn{4}{|c|}{$\gamma_{z z z z}^{\mathrm{nr}}(0 ; 0,0,0)$} \\
\hline & {$[\mu \alpha]^{0,0}$} & {$\left[\mu^{3}\right]^{I}$} & {$[\mu \alpha]^{I I}$} & {$\left[\mu^{3}\right]^{I I I}$} & {$\left[\alpha^{2}\right]^{0,0}$} & {$[\mu \beta]^{0,0}$} & {$\left[\mu^{2} \alpha\right]^{I}$} & {$\left[\mu^{4}\right]^{I I}$} \\
\hline I & $8.04 \times 10^{3}$ & $8.58 \times 10^{2}$ & $3.69 \times 10^{3}$ & $-3.23 \times 10^{4}$ & $1.02 \times 10^{6}$ & $8.49 \times 10^{5}$ & $6.24 \times 10^{5}$ & $3.94 \times 10^{4}$ \\
\hline II & $2.85 \times 10^{3}$ & $5.49 \times 10^{1}$ & $2.10 \times 10^{2}$ & $-9.13 \times 10^{2}$ & $2.27 \times 10^{5}$ & $3.23 \times 10^{5}$ & $1.11 \times 10^{5}$ & $-1.23 \times 10^{4}$ \\
\hline III & $3.79 \times 10^{4}$ & $2.77 \times 10^{4}$ & $-1.20 \times 10^{2}$ & $-1.79 \times 10^{4}$ & $2.80 \times 10^{6}$ & $-1.55 \times 10^{6}$ & $6.07 \times 10^{6}$ & $1.04 \times 10^{6}$ \\
\hline IV & $1.55 \times 10^{4}$ & $-1.41 \times 10^{4}$ & .. & $\ldots$ & $8.98 \times 10^{5}$ & $-1.24 \times 10^{7}$ & $-2.87 \times 10^{7}$ & $-2.18 \times 10^{7}$ \\
\hline V & $2.49 \times 10^{2}$ & $-1.60 \times 10^{2}$ & $1.87 \times 10^{1}$ & $1.11 \times 10^{2}$ & $1.63 \times 10^{4}$ & $-8.31 \times 10^{3}$ & $3.65 \times 10^{3}$ & $3.12 \times 10^{4}$ \\
\hline VI & $\ldots$ & $\ldots$ & $\ldots$ & $\ldots$ & $2.53 \times 10^{5}$ & $-1.16 \times 10^{5}$ & $3.64 \times 10^{5}$ & $-1.41 \times 10^{5}$ \\
\hline VII & $\ldots$ & $\ldots$ & $\ldots$ & $\ldots$ & $3.07 \times 10^{4}$ & $9.06 \times 10^{3}$ & $-1.16 \times 10^{3}$ & $-1.47 \times 10^{3}$ \\
\hline
\end{tabular}


the case of $\gamma^{\mathrm{nr}}(0 ; 0,0,0)$, the double harmonic approximation would have given an acceptable result only for molecules II and VII. For common dynamic processes only the OKE contains anharmonic contributions to the NR property under the infinite optical frequency approximation:

$$
\begin{aligned}
& \gamma_{z z z z}^{\mathrm{nr}}(-\omega ; \omega, 0,0)_{\omega \rightarrow \infty}=\frac{1}{3}\left[\alpha^{2}\right]_{z z z z ; \omega=0}^{0,0}+\frac{1}{2}[\mu \beta]_{z z z z ; \omega=0}^{0,0} \\
& \quad+\frac{1}{6}\left[\mu^{2} \alpha\right]_{z z z z ; \omega=0}^{I} .
\end{aligned}
$$

The fact that the anharmonic term in Eq. (29) has a smaller coefficient than the harmonic terms does not necessarily mean that anharmonicity is less important than for the corresponding static property because the two harmonic terms often occur with opposite sign. For the molecules considered in this paper there is a clear demarcation between I, II, and VII, which are affected to only a small extent by anharmonicity, and all the others which exhibit a large effect.

The C-ZPVA (hyper)polarizabilities contain only anharmonic terms. We have already examined the magnitude of these terms in comparison with the NR (hyper)polarizabilities. However, from our calculations it is not possible to provide a breakdown into square bracket types as we have done for the NR contribution in Table IX. This is because the analytical treatment of NR allows one to obtain [cf. Eq. (12)]:

$$
\gamma_{z z z z}^{\mathrm{nr}}(-\omega ; \omega,-\omega, \omega)_{\omega \rightarrow \infty}=\frac{2}{3}\left[\alpha^{2}\right]_{z z z z ; \omega=0}^{0,0},
$$

whereas the FF treatment of C-ZPVA does not yield the corresponding second-order square bracket term. On the other hand, a breakdown of the static $\beta^{\mathrm{c}-z p v a}$ into a sum of $[\mu \alpha]^{\mathrm{II}}$ and $\left[\mu^{3}\right]^{\mathrm{III}}$ terms is readily carried out.

The breakdown of the NR and C-ZPVA first hyperpolarizabilities presented in Table IX allows us to determine the initial convergence of the two contributing square bracket terms. In the case of $[\mu \alpha]$ the largest value of the ratio $[\mu \alpha]^{\mathrm{I} /} /[\mu \alpha]^{0,0}$ is 0.46 for molecule $\mathrm{I}$; otherwise it is less than 0.10 . On the other hand, the magnitude of $\left[\mu^{3}\right]^{\mathrm{III}} /\left[\mu^{3}\right]^{\mathrm{I}}$ is more than 10 for molecules I and II. For molecule V the magnitude of both of these ratios is less than unity but that is not true for $\beta^{\mathrm{c}-\text {-zva }} / \beta^{\text {nr }}$ because the two square bracket contributions to $\beta$ are of opposite sign for NR but have the same sign for C-ZPVA. This suggests that it may be more appropriate to compare individual square bracket terms than the total first (hyper)polarizability. That would lead to better initial convergence behavior as far as molecule $\mathrm{V}$ is concerned but not molecules I and II. Although we cannot make the same comparison for most of the square bracket terms that contribute to the second (hyper)polarizability we can do so for $[\mu \beta]$. The largest value of the ratio $[\mu \beta]^{\mathrm{II} /[\mu \beta]^{0,0}}$ is 0.66 for molecule I; otherwise it is less than 0.26 . Of course,

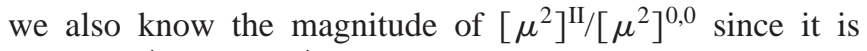
given by $\left|\alpha^{\mathrm{c}-z p v a} / \alpha^{\mathrm{nr}}\right|$. As discussed above, this ratio is always smaller than 0.11 , with the exception that in molecule I it is 1.2 .

\section{CONCLUSIONS AND FUTURE WORK}

$A b$ initio Hartree-Fock/6-31G and MP2/6-31G calculations of the longitudinal (hyper)polarizability - consisting of electronic (clamped nucleus), ZPVA, and pure vibrational
$(\mathrm{NR}+\mathrm{C}-\mathrm{ZPVA})$ contributions-have been carried out on a set of seven conjugated molecules that are representative of the various types often selected for their NLO properties. The pure vibrational contributions to the (hyper)polarizability may be classified according to their square bracket type and their total order in mechanical and electrical anharmonicity. Depending upon the square bracket type, the nonvanishing terms will be only even-order or only odd-order in the infinite optical frequency approximation, which we have used throughout. The NR vibrational (hyper)polarizability consists of all the lowest-order (nonvanishing) terms of each square bracket type. These go up to second-order for the static $\gamma$, first-order for the static $\beta$ and OKE; and zerothorder for the dc-P effect, dc-SHG, and intensity-dependent refractive index (IDRI). The next highest-order (nonvanishing) term of each square bracket type is included in the C-ZPVA (hyper)polarizability. Both the NR and C-ZPVA terms may be related to the change in equilibrium geometry induced by a static electric field. The effect of this geometry change on the static electronic electrical properties determines the NR contribution while the effect on the ZPVA correction to these properties determines the C-ZPVA contribution. Based on this perspective, one should monitor the initial convergence of the perturbation series, on the one hand, by comparing $P^{\text {zpva }}$ with $P^{e}$ and simultaneously, on the other hand, by comparing $P^{\mathrm{c}-\text { zpva }}$ with $P^{\mathrm{nr}}$.

Owing to computational difficulties many of the quantities required for comparison purposes have rarely, if ever, been previously determined for medium-size molecules at the MP2 level. Correlated ZPVA calculations have been limited to small molecules, whereas the C-ZPVA term has been reported only for molecules with no more than two heavy atoms. Although the NR (hyper)polarizability is obtained more frequently, only a couple of MP2 calculations beyond small molecules have appeared. With the aid of field-induced coordinates and, for C-ZPVA, a finite field technique, we have shown that the treatment of medium-size molecules is now feasible.

For individual molecules there are very substantial differences between the HF and MP2 results, not only for the absolute values of the various contributions to the (hyper)polarizability but also for their relative values. These differences are more pronounced, on the whole, for the ZPVA and C-ZPVA properties. Although our detailed analysis was carried out in terms of the MP2 calculations, we note that the general conclusions which emerge turn out to be similar with or without electron correlation taken into account. Only the 6-31G basis set was considered. It would be worthwhile in the future to study basis set extensions. On the basis of past experience we do not expect that such calculations will produce a significant change in the overall picture for the type of molecule considered here. However, this is a point that should be checked.

As in previous work, we have found that the NR term is commonly either larger in size than the static electronic (hyper)polarizability or comparable in size. This pertains to dynamic, as well as static NR properties. It may not be too surprising, then, that C-ZPVA (hyper)polarizabilities typically turn out to be larger than the ZPVA corrections. In 
addition, however, we find that static C-ZPVA hyperpolarizabilities are often larger than the corresponding static electronic property and that dynamic C-ZPVA contributions are often comparable in size to the latter. With regard to the ZPVA correction itself, the ratio $\left|P^{\text {zpva }} / P^{e}\right|$ (static property in either case) is usually small $(<0.13)$ but increases from $\alpha$ to $\beta$ to $\gamma$. For $\beta$ a maximum ratio of 0.37 was obtained in the case of molecule III; for $\gamma$ the maximum value, given again by molecule III, is 0.68 . Thus, the ZPVA term cannot always be neglected.

The behavior of the ratio $\left|P^{\mathrm{c}-z \mathrm{pva}} / P^{\mathrm{nr}}\right|$ differs between dynamic and static processes. For dynamic hyperpolarizabilities this ratio is always less than 0.68 for the molecules in our study and is typically much smaller. On the other hand, for static (hyper)polarizabilities we have found several instances where the ratio is greater than unity and quite a few others where it is too large to be neglected. As an alternative to the ratio of $P^{\mathrm{c}-\mathrm{zpva} / P^{\mathrm{nr}}}$ we examined the corresponding ratio for the individual square bracket terms that contribute to $P^{\mathrm{c}-\mathrm{pzva}}$ and $P^{\mathrm{nr}}$. Not all of these terms could be determined independently but, for those that could, we found their behavior to be similar to that described for the total property.

Our results indicate that higher-order terms in electrical and mechanical anharmonicity can make substantial contributions to the pure vibrational (hyper)polarizability of typical NLO molecules. For dynamic processes these contributions may be almost as important as the lowest-order terms; for static (hyper)polarizabilities they may be more important. Clearly, further work is necessary to characterize more specifically the circumstances that will lead to problematic behavior. One obvious possibility is that certain low-frequency vibrations are responsible. This issue and others can be explored by appropriately redefining the FICs that are used in our calculations. If a (very) limited number of critical vibrational motions can be identified, then it may be possible to treat them "exactly" without carrying out a perturbation expansion.

\section{ACKNOWLEDGMENTS}

One of the authors, M.T., thanks the Generalitat de Catalunya for financial help through CIRIT Project No. FI/0100699. The author, M.S., is indebted to the Department d'Universitats, Recerca i Societat de la Informació of the Generalitat de Catalunya for financial support through the Distinguished University Research Promotion 2001. Support for this work under Grant No. PB98-0457-C02-01 from the Dirección General de Enseñanza Superior e Investigación Científica y Técnica (MEC-Spain) is acknowledged.

${ }^{1}$ B. Champagne and B. Kirtman, in Handbook of Advanced Electronic and Photonic Materials, edited by H. S. Nalwa (Academic, San Diego, CA, 2001).
${ }^{2}$ D. M. Bishop and P. Norman, in Handbook of Advanced Electronic and Photonic Materials, edited by H. S. Nalwa (Academic, San Diego, CA, 2001).

${ }^{3}$ M. C. Magnoni, P. Mondini, M. Del Zoppo, C. Castiglioni, and G. Zerbi, J. Chem. Soc. Perkin Trans. 2 2, 1765 (1999).

${ }^{4}$ P. Macak, Y. Luo, P. Norman, and H. Agren, J. Chem. Phys. 113, 7055 (2000).

${ }^{5}$ B. Kirtman, B. Champagne, and J. M. Luis, J. Comput. Chem. 21, 1572 (2000).

${ }^{6}$ S. Millefiori and A. Alparone, Phys. Chem. Chem. Phys. 2, 2495 (2000).

${ }^{7}$ U. Eckart, V. E. Ingamells, M. G. Papadopoulos, and A. J. Sadlej, J. Chem. Phys. 114, 735 (2001).

${ }^{8}$ B. Kirtman and D. M. Bishop, Chem. Phys. Lett. 175, 601 (1990).

${ }^{9}$ D. M. Bishop and B. Kirtman, J. Chem. Phys. 95, 2646 (1991).

${ }^{10}$ D. M. Bishop and B. Kirtman, J. Chem. Phys. 97, 5255 (1992).

${ }^{11}$ D. M. Bishop, J. M. Luis, and B. Kirtman, J. Chem. Phys. 108, 10013 (1998).

${ }^{12}$ D. M. Bishop, B. Kirtman, and B. Champagne, J. Chem. Phys. 107, 5780 (1997).

${ }^{13}$ J. M. Luis, M. Duran, B. Champagne, and B. Kirtman, J. Chem. Phys. 113, 5203 (2000).

${ }^{14}$ J. M. Luis, M. Duran, and J. L. Andrés, J. Chem. Phys. 107, 1501 (1997).

${ }^{15}$ D. M. Bishop, M. Hasan, and B. Kirtman, J. Chem. Phys. 103, 4157 (1995).

${ }^{16}$ D. M. Bishop and E. K. Dalskov, J. Chem. Phys. 104, 1004 (1996).

${ }^{17}$ O. Quinet and B. Champagne, J. Chem. Phys. 109, 10594 (1998).

${ }^{18}$ J. M. Luis, M. Duran, and B. Kirtman, J. Chem. Phys. 115, 4473 (2001).

${ }^{19}$ J. M. Luis, J. Martí, M. Duran, J. L. Andrés, and B. Kirtman, J. Chem. Phys. 108, 4123 (1998).

${ }^{20}$ B. Champagne, J. M. Luis, M. Duran, J. L. Andrés, and B. Kirtman, J. Chem. Phys. 112, 1011 (2000).

${ }^{21}$ G. Papadopoulos, A. Willets, N. C. Handy, and A. E. Underhill, Mol. Phys. 88, 1063 (1996).

${ }^{22}$ D. Jacquemin, B. Champagne, E. A. Perpete, J. M. Luis, and B. Kirtman, J. Phys. Chem. (to be published).

${ }^{23}$ B. Kirtman, J. M. Luis, and D. M. Bishop, J. Chem. Phys. 108, 10008 (1998).

${ }^{24}$ J. M. Luis, B. Champagne, and B. Kirtman, Int. J. Quantum Chem. 80, 471 (2000).

${ }^{25}$ U. Eckart and A. J. Sadlej, Mol. Phys. 99, 735 (2001).

${ }^{26}$ D. M. Bishop, J. Pipin, and B. Kirtman, J. Chem. Phys. 102, 6778 (2001).

${ }^{27}$ J. M. Luis, M. Duran, J. L. Andrés, B. Champagne, and B. Kirtman, J. Chem. Phys. 111, 875 (1999).

${ }^{28}$ B. J. Krohn, W. C. Ermler, and C. W. Kern, J. Chem. Phys. 60, 22 (1974).

${ }^{29}$ E. A. Perpète, B. Champagne, J. M. André, and B. Kirtman, J. Mol. Struct. 425, 115 (1998).

${ }^{30}$ A sign error in equations (12), (13), (18), (19), (22), (23), and (25) in Ref. 13 has been corrected.

${ }^{31}$ A. D. Buckingham and W. Urland, Chem. Rev. 75, 113 (1975).

${ }^{32}$ The $E^{z p}$ form of Eq. (6) in Ref. 24 is correct only for $n=1$.

${ }^{33}$ D. M. Bishop, B. Champagne, and B. Kirtman, J. Chem. Phys. 109, 9987 (1998).

${ }^{34}$ M. J. Frisch, G. W. Trucks, H. B. Schlegel et al., GAUSSIAN 98, Revision A.10, Gaussian, Inc., Pittsburgh, PA, 1998.

${ }^{35}$ P. J. Davis and P. Rabinowitz Numerical Integration (Blaisdell, London, 1967), p. 166.

${ }^{36}$ W. J. Hehre, R. Ditchfield, and J. A. Pople, J. Chem. Phys. 56, 2257 (1972).

${ }^{37}$ D. Jacquemin, B. Champagne, and C. Hättig, Chem. Phys. Lett. 319, 327 (2000).

${ }^{38}$ B. Champagne, Élaboration de méthodes de chimie quantique pour l'évaluation des hyperpolarisabilités vibrationnelles-Conséquences pour l'optique non linéaire (PUN, Namur, 2001), pp. 68, 69. 